\title{
Small-Angle Scattering and Diffusion: Application to Relativistic Shock Acceleration
}

\author{
R.J. Protheroe, A. Meli* and A.-C. Donea \\ Department of Physics \& Mathematical Physics, The University of Adelaide, \\ Adelaide, SA 5005, Australia
}

19 June 2002

\begin{abstract}
We investigate ways of accurately simulating the propagation of energetic charged particles over small times where the standard Monte Carlo approximation to diffusive transport breaks down. We find that a small-angle scattering procedure with appropriately chosen step-lengths and scattering angles gives accurate results, and we apply this to the simulation of propagation upstream in relativistic shock acceleration.
\end{abstract}

Keywords: cosmic rays, diffusion theory, relativistic shock acceleration

\section{Introduction}

Relativistic charged particle transport in magnetized astro-physical plasma is strongly affected by magnetic irregularities, and may be approximated by diffusion. Diffusive transport of particles having speed $v$ can be simulated by a three-dimensional random walk with steps sampled from an exponential distribution with mean free path $\lambda=3 D / v$, where $D\left(\mathrm{~cm}^{2} \mathrm{~s}^{-1}\right)$ is the spatial diffusion coefficient, followed by largeangle (isotropic) scattering after each step (e.g. Chandrasekhar 1943), and this gives good results for distances much larger than $\lambda$.

In diffusive shock acceleration at relativistic shocks problems arise when simulating particle motion upstream of the shock because the particle speeds, $v$, and the shock speed $v_{\text {shock }}=c\left(1-1 / \gamma_{\text {shock }}^{2}\right)^{1 / 2}$ are both close to $c$, and so very small deflections are sufficient to cause a particle to re-cross the shock. Clearly, Monte Carlo simulation by a random walk with mean free path $\lambda$ and large-angle scattering is inappropriate here, and in Monte Carlo simulations of relativistic shock acceleration at parallel shocks Achterberg et al. (2001) consider instead the diffusion of a particle's direction for a given angular diffusion coefficient $D_{\theta}\left(\mathrm{rad}^{2} \mathrm{~s}^{-1}\right)$. Similarly, for a given spatial diffusion coefficient $D$, Protheroe (2001) and Meli \& Quenby (2001) adopt a random walk with a smaller mean free path, $\bar{\ell} \ll \lambda$, followed by scattering at each step by a small angle with mean deflection, $\bar{\theta}<1 / \gamma_{\text {shock }}$. See Bednarz \& Ostrowski (2001) for a recent review of relativistic shock acceleration.

* visiting from Imperial College, London

(C) 2018 Kluwer Academic Publishers. Printed in the Netherlands.

Protheroe.tex; 26/10/2018; 11:44; p.1 


\section{Small-angle scattering and diffusion}

We consider propagation by small steps sampled from an exponential distribution with mean $\bar{\ell} \ll \lambda$, followed at each step by scattering by a small angle sampled from an exponential distribution with mean $\bar{\theta} \ll \pi$. The change in direction $\left(\theta_{1}, \theta_{2}\right)$ may then be described as twodimensional diffusion with angular diffusion coefficient $D_{\theta}=\bar{\theta} v_{\theta} / 2$ $\left(\operatorname{rad}^{2} \mathrm{~s}^{-1}\right)$ where $v_{\theta}=\bar{\theta} / \bar{t}$, and $\bar{t}=\bar{\ell} / v$ such that $D_{\theta}=\bar{\theta}^{2} v /(2 \bar{\ell})$. The time $t_{\text {iso, }}$, which gives rise to a deflection equivalent to a large-angle (isotropic) scattering, is determined by $\left(\sigma_{\theta_{1}}^{2}+\sigma_{\theta_{2}}^{2}\right)^{1 / 2}=\sqrt{4 D_{\theta} t_{\text {iso }}} \sim$ $\pi / 2$, giving $\lambda \sim v t_{\text {iso }} \propto \bar{\ell} / \bar{\theta}^{2}$ and a spatial diffusion coefficient $D \propto$ $\bar{\ell} v / \bar{\theta}^{2} \propto v^{2} / D_{\theta}$. By using a Monte Carlo method it is straightforward to test this, determine the constant of proportionality, and thereby make the connection between diffusion and small angle scattering.

The solution of the diffusion equation for a delta-function source in position and time $q(\vec{r}, t)=\delta(\vec{r}) \delta(t)$ and an infinite diffusive medium is a three-dimensional gaussian with standard deviation $\sigma=\sqrt{2 D t}$ (Chandrasekhar 1943). The results from several Monte Carlo random walk simulations are shown in Fig. 1, from which we find that the expected dependence occurs for $\bar{\theta}<5^{\circ}$ at times $t>10^{5} \bar{\ell} / v$. For this case, we see from Fig. 1 that $\sigma^{2} \rightarrow 2 t v \bar{\ell} / 3 \bar{\theta}^{2}$, and so we obtain the connection between small-angle scattering and diffusion theory, namely $D \approx \bar{\ell} v /\left(3 \bar{\theta}^{2}\right) \approx v^{2} /\left(6 D_{\theta}\right)$.

\section{Application to relativistic shock acceleration}

As viewed in the frame of reference of the upstream plasma, ultrarelativistic particles are only able to cross the shock from downstream to upstream if the angle $\theta$ between their direction and the shock normal pointing upstream is $\theta<\sin ^{-1}\left(1 / \gamma_{\text {shock }}\right)$, where $\gamma_{\text {shock }}=(1-$ $\left.\beta_{\text {shock }}^{2}\right)^{-1 / 2}$ and $\beta_{\text {shock }}=v_{\text {shock }} / c$. For highly relativistic shocks these particles cross the shock from downstream to upstream travelling almost parallel to the shock normal. Similarly, having crossed the shock, only a very slight angular deflection, by $\sim 1 / \gamma_{\text {shock }}$ is sufficient to return them downstream of the shock. This change in particle direction gives rise to a change in particle energy $E^{\prime}$ and momentum $p^{\prime}$, measured in the downstream plasma frame (primed coordinates), of

$$
\frac{E_{n+1}^{\prime}}{E_{n}^{\prime}} \approx \frac{p_{n+1}^{\prime}}{p_{n}^{\prime}}=\frac{1-\beta_{12} \cos \theta_{n+1}}{1-\beta_{12} \cos \theta_{n}}
$$

in an acceleration cycle (downstream $\rightarrow$ upstream $\rightarrow$ downstream), where $\beta_{12}$ is the speed of the upstream plasma as viewed from the downstream frame. 


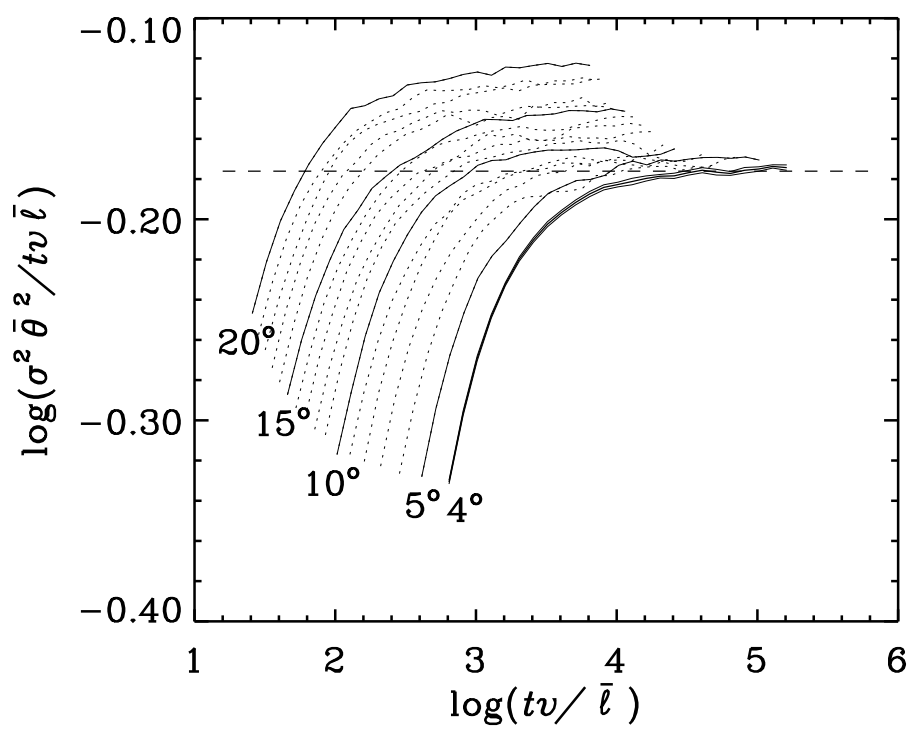

Figure 1. $\sigma^{2}$ vs. time for a $3 \mathrm{D}$ random walk with isotropic injection at the origin at $t=0$. Step-lengths $\ell$ were sampled from an exponential distribution with mean $\bar{\ell}$ followed by small-angle scattering with scattering angle $\theta$ sampled from an exponential distribution with mean $\bar{\theta}$ (the numbers attached to the curves). Dashed line is $\sigma^{2}=2 t v \bar{\ell} / 3 \bar{\theta}^{2}$. Curves for $5^{\circ}-20^{\circ}$ result from $10^{4}$ simulations; $4^{\circ}$ curve results from $8 \times 10^{4}$ simulations (width shows statistical error).

In "parallel shocks" the magnetic field is parallel to the shock normal, and so the the pitch angle $\psi$ is the angle to the shock normal and $v \cos \psi$ gives the component of velocity parallel to the shock. Thus the small-angle scattering method described in the previous section is used here to simulate particle motion upstream of a parallel relativistic shock, including the effects of pitch-angle scattering, for a given diffusion coefficient. We inject ultra-relativistic particles at the shock with downstream-frame energy $E_{0}^{\prime}$ travelling upstream parallel to the shock normal, i.e. $\theta_{0}=0$. We follow a particle's trajectory until the shock catches up with it, and it crosses from upstream to downstream with an upstream-frame angle $\theta_{1}$ to the shock normal and a downstreamframe energy $E_{1}^{\prime}$. The simulation was performed for $\gamma_{\text {shock }}=10$, and five different mean scattering angles $\bar{\theta}$, to determine the maximum $\bar{\theta}$ value that can safely be used for accurate simulation. The resulting distributions of $\cos \theta$ and $\log \left(E_{1}^{\prime} / E_{0}^{\prime}\right)$ are shown in Fig. 2, and show that in this application one requires $\bar{\theta}<0.1 / \gamma_{\text {shock }}$. Our results are quite consistent with those of Achterberg et al. (2001), who used a diffusive angular step $\Delta \theta_{\text {st }} \leq 0.1 / \gamma_{\text {shock }}$. 


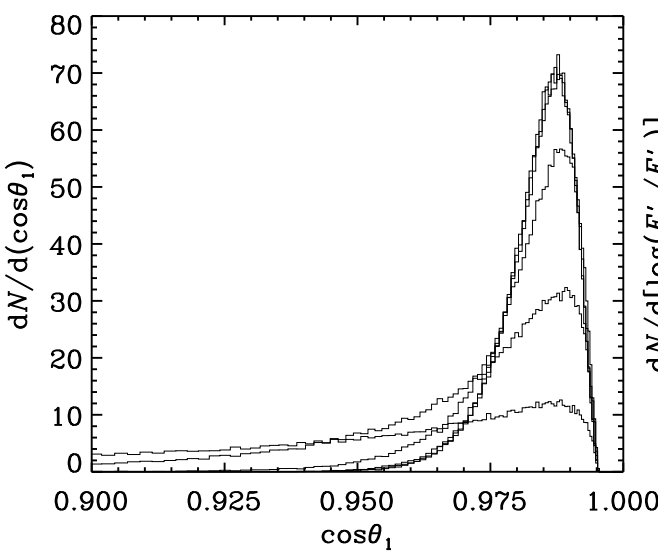

a. $\cos \theta_{1}$ distribution

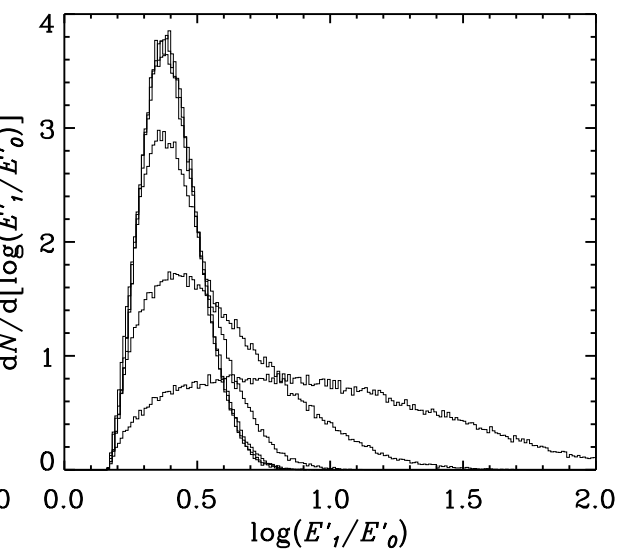

b. $\log \left(E_{1}^{\prime} / E_{0}^{\prime}\right)$ distribution

Figure 2. Small-angle scattering simulation of excursion upstream in diffusive shock acceleration at a parallel relativistic shock with $\gamma_{\text {shock }}=10$. Results are shown for $10^{5}$ injected particles and $\bar{\theta}=10^{-2} / \gamma_{\text {shock }}$ (top histogram), $3 \times 10^{-2} / \gamma_{\text {shock }}$, $10^{-1} / \gamma_{\text {shock }}, 0.3 / \gamma_{\text {shock }}, 1 / \gamma_{\text {shock }}$ and $3 / \gamma_{\text {shock }}$ (bottom histogram). Note that the top three histograms are almost indistinguishable.

\section{Conclusion}

The standard Monte Carlo random walk approach to simulation of energetic charged particle propagation for a given spatial diffusion coefficient $D$ can be extended to apply accurately to times much less than $\lambda / v=3 D / v^{2}$ by using a small-angle scattering procedure with steps sampled from an exponential distribution with mean free path $\bar{\ell}=\bar{\theta}^{2} \lambda$ followed at each step by scattering with angular steps sampled from an exponential distribution with mean scattering angle $\bar{\theta}<0.09 \mathrm{rad}\left(5^{\circ}\right)$. The spatial and angular diffusion coefficients are then $D \approx \bar{\ell} v /\left(3 \bar{\theta}^{2}\right)$ and $D_{\theta}=\bar{\theta}^{2} v /(2 \bar{\ell})$, and are related by $D \approx v^{2} /\left(6 D_{\theta}\right)$. In simulation of upstream propagation in relativistic shock acceleration one must use $\bar{\theta}<0.1 / \gamma_{\text {shock }}$ to obtain accurate results.

\section{References}

Achterberg, A., Gallant, Y.A., Kirk, J.G., and Guthmann, A.W.: 2001, MNRAS $328,393$.

Bednarz, J., and Ostrowski, M.: 2001, MNRAS 310, L13.

Chandrasekhar, S.: 1943, Rev. Mod. Phys. 15, 1.

Meli, A., and Quenby, J.: 2001, 27th Int. Cosmic Ray Conf. , Ed. M. Simon et al. vol. 7 , p. 2742 .

Protheroe, R.J.: 2001, 27th Int. Cosmic Ray Conf. , Ed. M. Simon et al. vol. 6, p. 2006, and p. 2014. 